Recherches en didactique des langues et des cultures

Les cahiers de l'Acedle

$4 \mid 2007$

Notions en questions : Méthodologies de recherche

\title{
Didactique des langues et recherche-action
}

Dominique Montagne-Macaire

\section{(2) OpenEdition}

\section{Journals}

Édition électronique

URL : http://journals.openedition.org/rdlc/5071

DOI : $10.4000 /$ rdlc.5071

ISSN : 1958-5772

Éditeur

ACEDLE

\section{Référence électronique}

Dominique Montagne-Macaire, "Didactique des langues et recherche-action », Recherches en

didactique des langues et des cultures [En ligne], 4 | 2007, mis en ligne le 21 août 2007, consulté le 11 septembre 2019. URL : http://journals.openedition.org/rdlc/5071 ; DOI : 10.4000/rdlc.5071

Ce document a été généré automatiquement le 11 septembre 2019.

\section{(†) $\ominus$

Recherches en didactique des langues et des cultures is licensed under a Creative Commons AttributionNonCommercial-NoDerivatives 4.0 International License 


\title{
Didactique des langues et recherche-action
}

\author{
Dominique Montagne-Macaire
}

\section{Introduction}

1 Fréquemment considérée comme simple instrumentalisation pragmatique, la rechercheaction ne jouit guère d'un statut très favorable dans le monde universitaire. On lui concède même parfois celui de parent pauvre de la recherche. La variété des courants qui la traversent, les multiples formes qu'elle prend, les nombreuses sciences qui s'en sont emparées ont certes contribué au flou qui règne sur la notion. Sa méthodologie, considérée comme intuitive, menée par les praticiens, et peu construite, ne la sert guère dans un contexte de recherche "à la française", soucieux de précision, de cartésianisme et d'apports quantitatifs. En outre, comme le relève Hugues Bazin (Bazin, 2003), il n'existe pas de validation en recherche-action, même s'il existe des validations d'acquis par la recherche-action. Bref, la recherche-action ne jouit pas d'une réputation de scientificité très solide dans notre pays. Elle est victime d'un déficit de clarification.

2 De nombreux travaux de type qualitatif utilisent cette forme de recherche pour peu qu'ils concernent les dispositifs éducatifs, au regard des pratiques, et comportent une part importante consacrée au changement (Van der Maren, 1999). En ce sens, la rechercheaction semble particulièrement appropriée pour bon nombre d'objets de la didactique des langues.

3 La recherche-action repose sur le principe d'un processus interventionniste conçu par ou à tout le moins avec les sujets impliqués et dont l'objectif est la modification par les praticiens de leur relation à leurs postures d'enseignement/ apprentissage, voire l'évolution de ces pratiques mêmes pouvant s'étaler sur une échelle allant d'une meilleure conscience d'elles à une plus grande maitrise, ou encore à une action sur elles en termes de modifications. Divers degrés de changement sont donc envisageables.

4 La recherche-action est ainsi présente dans une variété d'interventions allant de l'évaluation de dispositifs pour révision de programmes à l'intention de changer 
radicalement des pratiques ou comportements en passant par la résolution de problèmes. Le changement est pour Berbaum (Berbaum, 1982):

- soit spontané, comme celui de l'évaluation d'une action menée sur un terrain scolaire : par exemple la demande d'un regard extérieur formulé par une équipe de cycle pour valider une innovation pédagogique, telle des ateliers plurilingues en maternelle ou de nouveaux outils, comme Internet ;

- soit planifié, par exemple lors de la mise en place par un chef d'établissement (voire un recteur ou le Ministère de l'Education nationale) des cours en barrettes pour les langues en collège-lycée, pour insuffler une approche par compétences ou encore lorsqu'il s'agit de l'évaluation des dispositifs de langues vivantes en fin d'école élémentaire par la Direction de l'Evaluation et de la Prospective, pour assurer le lien entre l'école et le collège qui ont ensuite fourni les outils d'évaluation de Banqoutils ${ }^{1}$.

5 Selon le type de commande du changement: interne, provenant des praticiens, ou externe par exemple provenant d'un choix politique, le modèle de recherche-action peut varier. Dans la mesure où l'univers est changement, un état de non changement serait peu probable.

Qui dit changement, évoque un processus qui se réalise au nom de quelque chose, de valeurs la plupart du temps. Derrière la description du changement, se cachent ses fondements. Le rapport au changement interpelle l'éthique professionnelle de l'enseignant. Le changement renvoie aux conceptions des langues et de leur enseignement que portent les praticiens et les chercheurs.

7 Le changement ne se conçoit pas sans résistances ni frilosités. Sans routines figées ni certitudes, douleurs et deuils. Maîtriser le changement en didactique des langues, c'est vivre un processus en tensions permanentes, doser les acquis et le projet du devenir. C'est convoquer le sens des actions et sa propre responsabilité éthique.

\subsection{Clarification du concept de recherche-action}

- Dès lors, la présente communication va s'attacher à apporter une clarification de la notion même de recherche-action dans le domaine de la didactique des langues et des débats qui la constituent, et ce dans deux directions :

- l'une exploratoire, contributive à la description d'une méthodologie de la recherche-action, et à son explication ;

- l'autre contributive à la compréhension de la notion même, c'est-à-dire en référence aux théories scientifiques et aux valeurs qui la sous-tendent.

8 Ces deux dimensions renvoient à deux conceptions du monde complémentaires, bien que différentes. Berbaum, dans son approche de l'analyse systémique, oppose descriptionexplication d'une part et compréhension d'autre part. Il précise pour la compréhension :

Donner un sens à un comportement, c'est [...] le situer par rapport à d'autres comportements présents et passés, c'est dégager le projet auquel il correspond, c'est lui accorder une intentionnalité, une finalité. (Berbaum, 1982).

La description relève d'une tendance explicative de type naturaliste, qui recherche des lois, des liaisons entre variables, pouvant faire l'objet d'observations dans un environnement d'expérimentation. La description cherche les causes et mesure les effets. L'approche descriptive se veut rationnelle. 
10 La compréhension des phénomènes, elle, renvoie à une tendance plus subjective, celle de la quête de sens, mais également plus épistémologique, celle de la recherche des théories sur lesquelles assoir les interventions.

11 Alors que les deux dimensions s'opposent dans diverses méthodologies de recherche, la recherche-action, tout comme l'analyse systémique, les prend toutes deux en compte, en les considérant non comme antithétiques, mais comme complémentaires.

\subsection{Pratiques et recherche-action}

12 Nous poserons ici que la recherche-action est une posture de recherche pertinente pour aborder les pratiques d'enseignement/apprentissage en langues. En effet, issue de la pratique, elle vise à la compréhension de la complexité de cette dernière, pour reprendre les propos de Bernadette Charlier (Charlier, 1998). De plus, les langues étant des pratiques sociales et scolaires, la recherche-action s'offre tout naturellement comme une démarche pour observer leur enseignement/apprentissage en contexte institutionnel ou lors d'actions de formation.

13 A l'heure où la didactique des langues se tourne vers des objets de plus en plus complexes, elle doit également prendre en compte les réalités sociales des langues (la mondialisation, la variété des biographies langagières dans les sociétés civiles, etc.), les prescriptions des politiques linguistiques des états, eux-mêmes influencés par les politiques européennes et enfin les conditions de l'apprentissage et de l'enseignement des langues, c'est-à-dire à la fois des facteurs internes et externes au système scolaire, mais également des dimensions micro, macro et mésosociales.

14 La finalité de la recherche-action est d'intervenir sur les pratiques non pas exclusivement pour les modifier, mais afin de les rendre conscientes et les faire analyser et comprendre. Le changement n'est pas ici une révolution, il relève plutôt d'une évolution. Dans ses travaux sur la méthodologie de la recherche, Van der Maren décrit deux modèles de recherche-action qu'il classe en référence aux enjeux qu'ils portent : un enjeu centré sur l'action elle-même, c'est à dire à visée pragmatique et actionnelle, et un enjeu à visée politique.

\subsection{Qui bénéficie de la recherche-action?}

15 Le principe de toute recherche-action est qu'une action d'enseignement ou de formation peut être source de connaissance, réinvestie dans l'action en cours. Le bénéficiaire de l'action menée est alors l'action elle-même. Sont alors centraux le processus mis en œuvre et les interactions entre les composantes élémentaires du dispositif.

Cependant, l'action, qui vise à former des individus, à transformer la réalité, ou encore à décrire, voire à expliquer les situations sociales, comporte en elle-même une part de distance réflexive qui l'alimente. On connait les travaux de Donald Schön qui le premier a proposé le terme de praticien-réflexif pour les enseignants faisant acte de prise de distance réflexive par rapport à leurs pratiques et leurs postures d'enseignement (Schön, 1994). C'est le cas des ateliers d'analyse de pratiques mis en place dans les formations des jeunes enseignants de langues PE2 et PLC2. Les questionnements sont alimentés de savoirs théoriques, d'échanges entre participants et de retour sur les observations, jeux de rôles et récits de vie. Par exemple un enseignant (ou un groupe d'enseignants) qui choisit 
d'aborder lors d'un atelier d'analyse de pratiques la difficulté de la prise de parole des élèves à l'oral (l'une des questions les plus fréquentes en AAP, après celle de l'autorité), ne pourra faire l'économie de se questionner sur les théories de la communication, de regarder les apports sur les dynamiques de groupes et les interactions sociales, les représentations des langues et de leur apprentissage que véhiculent les élèves, les profils pédagogiques et de considérer les apports de la psychologie comme la dépendance du champ par exemple. Au plan de la langue, il devra connaître leur biographie langagière, vérifier que les compétences pragmatiques des élèves sont mobilisables (capacité à poser des questions, à réagir, à reformuler, etc.). C'est dans un va-et-vient entre pratique, recul et analyse, apports théoriques et ancrage dans un nouveau contexte que se fait l'apport et que se constituent les conditions favorables au changement. Le bénéficiaire de l'action peut alors être "soi-même", ou autrui, c'est-à-dire les praticiens, certains diraient les sujets (professeurs de langues stagiaires et formateurs) et/ou les chercheurs (groupe de pilotage par exemple). Sont alors directement impliqués, les représentations, les pratiques et comportements professionnels, les postures d'enseignement des acteurs. Le plus souvent, les deux axes, celui des personnes et celui des processus s'interpénètrent.

En revanche, il n'y a pas deux recherches-actions qui se déroulent de manière identique, car celles-ci sont éminemment flexibles et fonction du contexte.

Nous dirons donc à ce stade qu'une recherche-action en didactique des langues est une intervention sur un dispositif d'enseignement/apprentissage ou de formation/ accompagnement effectuée par un individu ou par un groupe, accompagné par un ou plusieurs chercheurs, menée de manière collaborative, et dont le changement constitue l'un des axes majeurs.

Enfin une recherche-action s'inscrit dans le temps et dure rarement plus de trois ans, eu égard au fait qu'elle sollicite fortement les enseignants en dehors de leurs pratiques.

Une recherche-action débouche sur un produit validé, que ce soit dans un contexte isolé, ou mieux, de manière large. Le produit pouvant être une tâche, un dispositif, une publication, un rapport, une manifestation, des outils de cours, etc. Cette étape garantit la possibilité du transfert parce que les acteurs se sont situés dans le cadre de l'évaluation de leur intervention.

\section{Aperçu historique et tendances pour notre propos}

\subsection{La naissance du concept de recherche-action}

21 Un bref aperçu historique permet de cerner quelques aspects de la notion de rechercheaction telle qu'elle nous intéresse aujourd'hui en $\mathrm{DDL}^{2}$.

On attribue l'invention du terme recherche-action à un anthropologue, Collier (Collier, 1945). Celui-ci menait des recherches sur les indiens d'Amérique dans les réserves et proposa que ses découvertes soient utilisées au profit d'une politique favorable à ces derniers. De cette première apparition du terme, on retiendra une dimension sociale et une intervention sur une réalité observée que l'on souhaite modifier de l'extérieur pour une réalité visée et considérée comme valide en référence à des valeurs.

Historiquement, la recherche-action voit le jour aux Etats-Unis dans l'immédiat aprèsguerre, qui doit faire face à un nombre grandissant de problèmes : ghettoïsation, racisme, paupérisation et industrialisation massive, suites de la guerre, pénurie alimentaire, etc. 
Elle se développe dans les années 50 avec, selon Hugues Bazin (Bazin reprenant et remaniant deux versions d'un texte de Georges Lapassade, 1991-93³), un décalage pour l'Europe. L'Ecole de Chicago propose alors une voie de recherche originale et implicationnelle (Barbier, 1996) en instituant les Life stories, les récits de vie ou histoires de vie, qui révolutionnent les approches quantitative européennes, faites d'enquêtes, de questionnaires et de statistiques hérités de Durkheim. A cette époque, les chercheurs, sociologues, s'appuient sur des relais dans les communautés qu'ils étudient. Avec ces praticiens, ils élaborent de manière concertée des connaissances qui viennent du terrain et y retournent, des propositions de solutions pour des changements possibles. La volonté de résoudre des problèmes rencontrés dans la société américaine s'adosse à l'idée que les acteurs des communautés eux-mêmes peuvent prendre appui sur leurs propres actions en tant que groupe, et produire du changement, d'attitudes, de comportements.

La recherche-action est largement issue des théorisations du psychologue allemand Kurt Lewin (Lewin, 1943), émigré aux Etats-Unis en 1934, et qui développa entre autres la théorie de la dynamique des groupes, avec une échelle de degrés d'implication du chercheur plus ou moins importants. Selon Dominique Guénard (Guénard, 1991) :

Vers la fin des années 30 , les pouvoirs publics américains ont donné pour mandat à Lewin de modifier les habitudes alimentaires de la population. Il a élaboré alors les concepts de la dynamique des groupes, terme qu'il a créé pour désigner les processus qui régissent le fonctionnement des groupes. [...]Lewin a tenu à ce que théorie et pratique soient méthodologiquement liées. Cette philosophie a donné naissance au concept de recherche-action.

Si Kurt Lewin a porté une attention toute particulière aux phénomènes de groupes humains socialement constitués, il a en particulier mené des expériences dans l'institution scolaire. Au cours de l'une d'entre elles, il étudie trois ambiances et le comportement d'apprenants dans trois situations de pouvoir : l'une autocratique, l'autre démocratique et la troisième celle du laisser-faire. A la fin de l'enseignement, les élèves sont invités à disposer de leurs productions. Ceux du groupe autocratique détruisent leur œuvre. Ceux du groupe démocratique offrent leurs créations à leurs camarades, ceux du groupe dit du laisser-faire en font un feu de joie. Lewin pensait possible pour le chercheur d'accompagner des organisations sociales d'un fonctionnement autocratique vers un fonctionnement démocratique et participatif. Au plan théorique, Kurt Lewin se rapproche de la Gestalt Theorie, la théorie psychologie de la forme, dont les représentants sont Wertheimer, Köhler, Koffka, et en France Guillaume, Merleau-Ponty, et dans laquelle le tout est plus que la somme des parties qui le constituent, il est même perçu avant les parties. Ce qui amène Kurt Lewin à des analyses plus globales des comportements humains, considérés comme des ensembles, accordant une importance forte aux contextes (le champ psychologique et social) dans lesquels les individus agissent et le groupe vit ses représentations et ses actes. S'il est l'un des initiateurs des théories de l'intersubjectivité humaine et des résolutions de problèmes communicationnels, il est également considéré comme l'un des auteurs des théories de l'organisation et du management.

Lewin écrit à propos de la recherche-action :

The research needed for social practice can best be characterized as research for social management or social engineering. Is it a type of action-research, a comparative research on the conditions and effects of various forms of social action, and research leading to social action. Research that produces nothing but books will not suffice (Lewin, 1948). 
Bien que les idées de Kurt Lewin aient abondamment alimenté les travaux sur la recherche-action, on leur attribue cependant quelques faiblesses. Lewin portait peu d'attention aux phénomènes inconscients, y compris les inconscients collectifs. Il proférait une foi inébranlable dans la science et le progrès humain. Un dernier reproche lui a souvent été fait de minimiser les conflits de classe sociales, notamment dans sa méthode de résolution des conflits ethniques, qui fut un échec.

Les années 60 ont vu l'action-research décliner, du fait de ses liens avec les idéologies politiques (l'activisme politique radical aux USA, par exemple), en Grande-Bretagne, en France comme au Canada. Cette radicalisation se définit selon René Barbier comme :

un changement qui résulte d'une transformation de l'attitude philosophique du chercheur concerné à l'égard de son propre rapport au monde (Barbier, 1996).

A cette époque, la recherche-action part en quête de valeurs, de sens et d'un questionnement épistémologique. Elle ré-interroge les sciences humaines, se refusant à n'être qu'une nouvelle méthodologie, qui ne remet en rien en cause l'ordre dominant de la scientificité (Barbier, 1996).

30 Par la suite, les années 70-80 contribuent à rationaliser l'approche. On revient alors de manière insistante sur le fait que l'action-research est collaborative et orientée vers les pratiques (Carr et Kemmis, 1986; Kemmis et Mc Taggart, 1988). Les travaux de groupes voient leurs heures de gloire annoncées. Ce que développe Mc Taggart en ces termes :

Action-research is not a method or a procedure for research but a series of commitments to observe and problematize through practice, a series of principles for conducting social enquiry. (Mc Taggart, 1996

\subsection{La recherche-action en France}

31 Après une apparition en Europe dans les années 50, puis un net déclin, la rechercheaction revient dans le domaine de l'enseignement. Le champ des sciences de l'éducation, voué à la formation et à l'apprentissage scolaire, occupe largement le terrain de la recherche-action à partir des grandes réformes de l'école dans les années 80 , au travers d'études en sociologie (Crezé et Liu, 2006; Mesnier et Missotte, 2003), en psychologie sociale (Levy, 1997), en sociologie de l'intervention (Vrancken et Kuty, 2001) ou en ethnographie (Lapassade, 1993), etc.

La recherche-action en France connait alors de profondes évolutions, s'éloignant des perspectives initiales de Kurt Lewin, et se rapprochant :

- pour une part d'approches cliniques (Blanchard-Laville, 2003); Ces approches ont livré des observations des pratiques enseignantes, des travaux sur l'analyse de pratiques, par exemple, les contrats et régulations didactiques, l'espace psychique de la classe ;

- pour une autre part d'approches sociologiques (Barbier, 1996 ; Bazin, 2003 ; Amado et Levy, 2001), qui fournissent des travaux sur les interactions, les postures et rôles des acteurs, les approches par tâches ${ }^{5}$.

- Dans un tour d'horizon des différents types de recherche-action contemporaines, René Barbier propose la classification suivante :

- les recherches-action d'inspiration lewinienne ou post-lewinienne : oeuvrant sur un terrain réel, impliquant des acteurs de ce même terrain, le chercheur à l'initiative de la recherche est confronté à un problème concret dans lequel il doit s'impliquer pour avancer ; 
- la consultation-recherche d'inspiration analytique ou socioanalytique: le chercheur agit comme thérapeute, à la demande des acteurs. Le changement attendu est d'ordre sociothérapeutique ;

- l'action-recherche : destinée à favoriser des recherches en vue de changements volontaires décidés par le chercheur ; ces changements ont été au préalable formulés par le groupe des acteurs au terme d'une recherche menée sur eux-mêmes ; il y a recherche et co-action ;

- l'expérimentation sociale, ou expérimentalités sociales pour Barbier; ce sont des formes de recherches en actes, des expériences de vie qui engagent l'être et son devenir, mettent à l'épreuve des idées, les soumettent à l'analyse.

\subsection{Recherche-action et didactique des disciplines}


grammaticale en allemand, d'un travail en pédagogie de projet en anglais au premier degré, de la mise en ateliers multisensoriels d'enfants de maternelle, de la quête de meilleures compétences phonologiques dans une langue donnée, etc. De tels dispositifs existent ou du moins leur cadre, et sont amenés à évoluer grâce à la recherche-action. Ils s'inscrivent dans la durée. Les acteurs sont plus ou moins cobayes, et plus ou moins conscients des objectifs poursuivis ;

- des actions de formation d'enseignants de langues et d'accompagnement d'équipes et des projets dans des établissements ou des régions. C'est le cas par exemple lors des formations des futurs professeurs des écoles amenés à gérer un projet langues au cycle III, ou encore la réflexion en équipe de professeurs de langues sur la mise en place des cours en barrettes dans un établissement pour favoriser le travail par compétences en interlangue. Ces actions s'inscrivent dans une durée plus ou moins brève à laquelle on se consacre pleinement, les acteurs en sont généralement volontaires et marquent leur plus ou moins grande adhésion. Enfin, les sujets ont la conscience de l'existence de l'action de formation, même s'ils n'ont pas une conscience très nette des objectifs poursuivis (Berbaum, 1982) ;

- des actions de recherche portant sur des innovations pédagogiques et didactiques, comme le programme européen d'éveil à la diversité des langues et des cultures Evlang ou le dispositif franco-allemand de rencontres numériques par visioconférence, développant des compétences interculturelles et pragmatiques (oral), tele-tandem. De tels dispositifs n'existent pas ou peu dans les pratiques des partenaires de la recherche-action au moment de leur conception. Ils résultent d'une commande externe dans les cas cités, mais peuvent être initiés de manière interne. Ils s'inscrivent dans un espace de création.

Constituée de deux composantes, la recherche et l'action, la recherche-action tire sa légitimité de l'articulation (harmonieuse) des deux. La démarche devient opérante lorsque tombe la dichotomie entre recherche et action. La recherche-action a des effets tant sur les pratiques que sur les théories qu'elle alimente.

Nous distinguerons la recherche-action dans un cadre d'intervention de la rechercheaction dans un espace de création, selon que la commande repose sur une invention, une innovation ou une intervention de type évaluation ou ajustement.

Enfin, la recherche-action en didactique des langues peut souvent être considérée comme un système ouvert et non fermé, c'est-à-dire comme un espace de création et non comme un simple champ d'intervention. Un système ouvert offre la possibilité d'apports complémentaires, voire d'hybridations. Le pari d'une telle conception repose sur la conviction que les praticiens peuvent travailler de manière autonome.

De nouveaux objets apparaissent en lien avec les pratiques enseignantes comme le processus de conception-prodution-diffusion d'un manuel de langues à partir de la place occupée par les auteurs et le chercheur dans une action située. L'action part de l'équipe conceptrice, faite des sensibilités des auteurs et des éditeurs, elle répond à un besoin et à une demande. Elle est de nature top down. Le choix des référents théoriques s'avère vital pour l'équipe. Tous doivent partager les mêmes référents ou comprendre ceux des autres partenaires. Ce sont ces référents qui alimentent l'analyse de départ des concepteurs. Ces derniers se trouvent dans une situation complexe dans la quelle ils doivent prendre en compte à la fois :

- les attentes éditoriales (d'ordre financières et budgétaires)

- les demandes des enseignants (didactiques et pédagogiques)

- leurs savoirs d'expérience

- les contraintes temporelles ou spatiales de l'action 
- les théories de référence en didactique de l'acquisition d'une L2

- les programmes officiels et la didactique institutionnelle

- leurs facultés de créativité pour se démarquer de l'existant

1946 ; Caillot, 2005 ; Kemmis et Mc Taggert, 1988, repris par Mc Isaac, 1996 ; Susman, 1983) montrent un principe de cercles répétitifs, reprenant un schéma identique. Pour Lewin et les autres chercheurs, on passe en spirales successives de l'observation d'un champ d'action, dont on tire une situation-problème, à son analyse en contexte qui aboutit à un plan d'action. Ce plan d'action est étudié à son tour, des ajustements éventuels sont proposés, une réflexion est menée sur les effets de l'action, s'il y en a eu, puis en découle une nouvelle planification, suivie à son tour d'un cycle complet aboutissant à une évaluation. Le schéma est ainsi en boucle, fonctionne par cycles brefs, dont la fréquence garantit une certaine efficacité.

On distingue généralement cinq phases constitutives de la méthodologie de la rechercheaction : la phase diagnostic de la situation problème, la prise de décision des praticiens et des chercheurs sur les actions à mener qui débouche sur un plan d'action, l'analyse postérieure à l'action des effets et ajustements par rapport à des incidents critiques, la phase d'évaluation et une phase de rétroaction et transfert. Nous allons revenir sur ces phases. Elles correspondent à divers registres d'accompagnement d'équipes par exemple.

\subsection{Critères caractéristiques d'une intervention}

Un certain nombre de conditions doivent être réunies pour qu'une action-recherche soit efficace. Il s'agit des critères suivants :

- il faut une situation de départ susceptible d'être problématisée et diagnostiquée comme telle, car c'est ainsi qu'elle acquière un statut pour la recherche. Le problème soulevé se doit d'être en lien avec le changement visé ;

- il faut qu'existe un groupe social d'acteurs (praticiens et chercheurs), plus ou moins unis, plus ou moins conscients de cette problématique, mais en mesure de résoudre le problème par une action collective, négociée et flexible, proche de la création (faisant appel à leurs compétences, intuitions et connaissances acquises ou à construire), praticiens plus ou moins 
engagés qui deviennent des praticiens chercheurs pour certaines obédiences, ou qui s'associent avec des chercheurs pour d'autres écoles. La recherche-action est une intervention collaborative favorable au travail d'équipe ;

- il faut que puissent être vécues des pratiques réflexives régulières en particulier pour évaluer les décisions et mesurer les effets de l'action. Ces pratiques s'appuient sur la négociation, l'explicitation, l'élucidation, la justification, l'exploration et la validation d'hypothèses, la formulation de pistes et de propositions. Elles se rapprochent de l'entretien d'explicitation et d'autres approches d'analyse réflexive (Schön, 1987, Paquet et alii, 1996). La littérature du domaine a développé la notion d'incident critique (Altet, M., 1996, Jonnaert et Van der Borght, 1999, Newman, 2000). Ces incidents critiques permettent d'analyser ses pratiques, c'est-à-dire d'en prendre conscience, voire de les transformer dans certains cas ;

- il faut des produits et des traces écrites qui permettent d'analyser, de réguler et d'évaluer l'action dans son plan et dans sa réalisation. C'est dans ce sens que sont réunis des comptesrendus de réunions, des scripts d'enregistrements sonores, des vidéogrammes, des journaux de bord, des préparations de cours, des productions d'élèves, des parcours et histoires de vie, des enregistrements de manipulations de claviers d'ordinateurs et de souris, etc. En outre, une certaine rigueur est attendue pour collecter les données et les analyser. On utilisera plusieurs types d'outils issus des approches qualitatives, comme les entretiens, les observations (parfois participantes), les études de cas, les monographies, les comptes-rendus de réunions sous forme de textes, mémos, notes de synthèse, photos, etc. Le croisement des outils sera effectué de manière rigoureuse. On se référera ici utilement à l'ouvrage de Van der Maren, pour les aspects méthodologiques précis (Van der Maren, 1999) ;

- il faut qu'existe une possibilité de décalque vers d'autres situations ou de nouvelles créations. Ceci passe par la valorisation de la recherche-action et la communication de ses conclusions, première étape de formalisation. Mais aussi par la recherche d'expériences proches et/ou de théories explicatives pouvant enrichir le nouveau questionnement et favoriser des échanges de pratiques.

\subsection{Niveaux d'élaboration des interventions}

Suivant en cela les travaux d'Ardoino (Ardoino, 1966), rappelés par Roegiers (1997), on considère généralement qu'existent cinq niveaux d'intervention en recherche-action, même si ces niveaux, tout comme précédemment, sont amenés à interagir, à subir des ajustements respectifs dans leurs liens et tensions :

- le niveau des personnes ou niveau intrapsychique: il relève des caractéristiques personnelles et professionnelles. C'est à ce niveau que se joue le travail sur les représentations de l'objet d'apprentissage ou du métier d'apprenant ou du métier d'enseignant en formation par exemple. Ou encore l'auto-socio-construction des apprentissages en langues, comme en termes de découverte de son profil d'apprentissage ou de son style d'enseignement. Gather Thurler écrit à ce propos :

La théorie de l'apprentissage organisationnel admet que les savoirs mobilisés dans l'action individuelle sont dans une large mesure socialement déterminés. En même temps, elle soutient que l'individu a une certaine prise sur la transformation de ces surdéterminations cognitives. L'action pouvant déclencher un processus de mise à l'épreuve et de révision des cognitifs construits. (Gather Thurler, 2000);

- le niveau des interrelations: ce niveau analyse les actions, réactions, interactions entre acteurs, particulièrement sensible dans le registre des théories de la communication et l'acquisition des langues ; 
- le niveau du groupe ou niveau microsocial : que ce soit le groupe d'apprenants, celui des enseignants en formation, des équipes pédagogiques constituées lors d'un accompagnement, dans un établissement. Ce groupe, considéré comme sujet, fonctionne par rapport à ses besoins et rôles avec une dynamique qui lui est propre ;

- le niveau mésosocial, ou niveau intermédiaire : celui des organisations et des fonctions par rapport aux missions attendues. C'est le niveau des établissements, des régions, des lieux et instances stratégiques pour des décisions locales ;

- le niveau macrosocial ou institutionnel: c'est le niveau de la société, des théories de référence, des valeurs, des finalités. A ce niveau, souvent articulé avec une strate européenne, se joue la politique des langues d'un état. Il s'agit d'un niveau largement prescriptif (ex.: les programmes officiels), plus rarement incitatif (ex.: Documents du Conseil de l'Europe comme le Cadre européen commun de référence pour les langues vivantes).

La recherche-action peut concerner plusieurs niveaux à la fois. Pour rendre compte de la complexité, elle doit à la fois faire émerger et analyser les divers niveaux et les mettre en relation par le biais des liens et tensions qui les animent, des ajustements qui les caractérisent. Prenons comme exemple le plan de formation des Professeurs des écoles, qui a été modifié en 2006-2007, ce qui a provoqué une grave inquiétude chez les formateurs qui l'ont découvert tardivement, en juin 2006. Lors d'un travail exploratoire, chacun d'eux a été amené à énoncer ses propres conceptions de la formation, ses attentes et ses frustrations (nombre d'heures, spécificité de la discipline, etc.). Puis dans un second temps, ont été clarifiées les attentes de l'institution, c'est-à-dire les commandes du Ministère (réduction des horaires de manière drastique : la moitié des heures accordée, suppression des dominantes langues vivantes, centration exclusive du concours de recrutement sur les compétences linguistiques des candidats, etc.). On a ensuite présenté l'espace de liberté, négocié au niveau mésosocial avec la direction de l'Institut, que l'on a distingué des exigences du Ministère, placées, elles, au niveau macrosocial. Puis le groupe a travaillé sur la caractérisation de certaines situations-problèmes et de représentations comme l'idée qu'on ne produit pas des linguistes bilingues en formation, mais des professionnels de la gestion de projets plurilingues et de l'enseignement de l'une d'entre elles, et que cela s'accompagne de compétences à définir, puis dont il convient de se doter. Enfin, il a élaboré des modèle de formation prenant en compte les apports de la recherche (comme l'apport de la psycholinguistique sur l'acquisition d'une L2 après une L1 ou plusieurs L1 entre 8 et 12 ans ou la clarification des théories de l'apprentissage d'une L2 et le modèle de la socio-construction), apports considérés comme essentiels à la gestion des apprentissages et à la formation professionnelle. Dans le modèle, se trouvent également placés les savoirs d'expérience des formateurs, les résultats des étudiants ayant passé l'épreuve de langues au concours, et les retours des anciens sur leurs premières expériences. Parmi les dispositifs, ont alors été proposés des modules de formation interlangues avec des temps spécifiques à l'une d'entre elles, organisés selon les centres d'intérêt des stagiaires et les compétences des formateurs. Sont ainsi nés des parcours à géométrie variable, basés sur le minimum horaire en présentiel respectant les attentes du Ministère, complété de temps en autonomie (par exemple pour une mise à niveau linguistique) ou en tutorat direct voire à distance (pour les retours de pratiques et d'expérimentation) et sur serveur (pour des documents interactifs, des vidéos à la demande, diffusées en streaming par exemple). L'un des premiers effets de ce choix de parcours de formation est la mise en équipe des formateurs, des échanges plus fréquents entre eux, concernant les savoirs savant plus que les savoirs d'expérience, donc une 
progressive objectivation des discours, une plus grande autonomie dans les projets des stagiaires, une augmentation des propositions émanant d'eux en termes d'ajustements, un véritable réseautage et un regard davantage plurilingue chez les formés, ce qui constitue une manière de répondre à la sollicitation de Véronique Castellotti, lorsque celle-ci écrit :

Il ne s'agit pas seulement de former des enseignants à la pluralité mais aussi principalement par la pluralité, en imaginant des scénarios susceptibles de leur faire vivre la diversité linguistique et culturelle. (Castellotti, 2006).

Ce modèle de formation a été validé par la direction et contractualisé dans la brochure des étudiants et stagiaires de l'Institut. Il bénéficie d'un protocole de suivi et d'évaluation en début, en cours et en fin d'année universitaire pour les formateurs et les formés. Pour revenir brièvement sur les niveaux précités en référence à Ardoino, le plan de formation concerne les trois derniers.

\subsection{Les contextes et les acteurs}

51 La connaissance du contexte et des autres acteurs du système éducatif est nécessaire pour agir. Chaque environnement étant unique, les professionnels des langues doivent également être de bons analystes des situations qu'ils rencontrent et des contextes dans lesquels ils agissent. Si tel établissement a une solide culture des notes, des contrôles fréquents, placés sur des plages horaires balisées pour des tests réguliers, avec des bulletins de notes et des demandes de signature des contrôles par les parents, bref, dans ce type de système, on ne pourra guère imaginer la suppression des notes et le passage à l'auto-positionnement et l'auto-évaluation, même si intellectuellement les enseignants le désirent. Quelques étapes précédentes seront nécessaires. On peut dire que la culture pédagogique d'un établissement engendre certains modes de fonctionnement plutôt que d'autres et peut même valoriser des pratiques ou des acteurs (les parents par exemple) a détriment d'autres.

De la même manière, les lieux pour apprendre les langues se multiplient : salle de classe, espace polyvalent, cour de récréation, centre de documentation, salle informatique, amphithéâtre, en itinérant avec des I-pods, etc. De plus en plus l'accès au savoir se joue dans et en dehors du lieu "classe"7, à sa périphérie. A ces divers lieux correspondent de nouvelles modalités de groupement des élèves (par binômes, en groupes, seuls...) et d'interactions tout autant que de nouvelles manières d'accéder au savoir.

De ces contextes, régulièrement mis en avant comme des obstacles par les praticiens, on peut retenir en suivant Bazin que c'est la situation qui constitue l'unité de sens d'une recherche-action (Bazin, 2003). Etre en recherche-action, c'est être en situation, celle-ci étant largement opérationnalisée par les praticiens eux-mêmes.

\subsection{La posture du chercheur}

54 L'une des difficultés de la recherche-action réside dans la possible confusion des rôles entre et les chercheurs et les praticiens. On aurait d'un côté la question du Comment faire? et de l'autre celle du Pour quoi faire? La dichotomie est levée dès lors que les rôles de chacun sont explicites. De plus, l'on constate de ce fait une meilleure organisation du travail et une plus grande efficacité dans le respect des plannings. 
55 La recherche-action revisite le schéma linéaire, entre-temps classique, de production diffusion consommation du savoir, en intégrant les praticiens (dans notre cas, le plus souvent les enseignants et étudiants ou stagiaires professeurs de langues et professeurs des écoles) dans le processus de production du savoir. Ceux-ci, en situation, sont amenés à produire des connaissances qui nourrissent la recherche en retour. C'est le cas des étudiants de Master Pro2 qui alimentent leur groupe de pairs et la recherche par des échanges et des productions d'outils spécifiques. Ou celui des valises pédagogiques multimédia des PE2 qui, outre l'intérêt qu'elles présentent pour leurs auteurs, constituent une somme à disposition pour les générations futures autant que des outils d'analyse pour la recherche.

Enfin, si la double posture du chercheur, entre implication et observation, peut prêter à confusion, c'est peut-être parce que deux conceptions de la recherche et des actions d'intervention sont à l'œuvre: le chercheur impliqué renvoie aux approches ethnographiques (Lapassade, 1993). Le chercheur qui adopte une posture distanciée se place dans une lecture moins engagée, voire moins militante de l'intervention. Il est moins modélisant.

Quoi qu'il en soit, le rôle du chercheur, en tant que responsable, consiste à mettre en place la recherche-action, à alimenter ses cycles brefs, à faire réagir les praticiens sur le terrain en tant que catalyseur, à les impliquer, en tant que stimulant, à les observer en croisant divers outils et à leur apporter des éléments nouveaux issus de la recherche. Il gère également le passage à la trace écrite et à la mémoire du projet. C'est souvent lui qui rédige le rapport final et conceptualise les travaux de l'équipe.

Une question d'éthique est nécessairement soulevée. Tous les praticiens ne sont pas demandeurs, tous n'adhèrent pas aux méthodes de la recherche-action, ce qui renvoie à la liberté de décision de chacun. Un lien de confiance doit s'établir. Il n'est pas rare que des approches plus personnelles favorisent la détente et la contribution des réticents. Mais jusqu'où peut ou veut bien aller le chercheur? Le respect de tous s'impose. Ce respect et cette tolérance se vivent dans la communication ou dans l'anonymisation des données. On en se cachera pas le fait que le chercheur a au départ un statut dominant du fait de son rapport au savoir (il arrive comme expert et est souvent perçu comme modèle).

\section{La dimension modélisante dans une recherche- action}

Deux aspects attirent ici l'attention :

- celui du protocole de recueil de données et de la conduite de l'intervention;

- celui de la duplication de la recherche-action.

60 La recherche-action est une démarche qualitative plus que quantitative. Elle reprend les outils spécifiques de son mode de fonctionnement, à savoir des outils de communication directe: entretiens semi-directifs, observations participantes. Elle s'appuie sur la convivialité, en allégeant les dispositifs ou en les rendant plus ludiques : travail avec des enregistrements vidéo, à partir de photos, activités de dessin, etc. Elle doit souvent être réactive aux incidents critiques, s'adapter et reste de ce fait largement empirique. 
61 Néanmoins, pour avoir valeur de recherche, la recherche-action repose sur un protocole précis qui croise les outils de recueil de données et s'inscrit dans une durée bien délimitée et gérable.

62 Si les résultats d'une intervention renseignent bien sur le contexte précis dans lequel s'est joué la scène, il n'est pas toujours possible de vérifier la transférabilité des analyses à d'autres cas. On validera au moins le cas étudié et ce de manière exhaustive. Ce qui fait écrire à Catroux que l'authenticité peut alors servir de substitut à la validation des conclusions. (Catroux, 2002).

63 La question centrale qui sous-tend la réflexion sur la recherche-action est celle de sa duplication. Les changements sont-ils stables au bout d'un certain temps ? Comment s'y prendre pour qu'ils soient durables? Peut-on enseigner des stratégies opératoires, Des vigilances ? Peut-on imaginer un modèle d'accompagnement du changement ?

Etant donné la variété des situations et la spécificité des contextes rencontrés, d'autant qu'il s'agit d'œuvrer sur la matière humaine, il est réaliste de se demander si le transfert des acquis d'une recherche-action est possible à d'autres situations d'enseignement ou de formation que la situation particulière abordée et à d'autres personnes que celles directement impliquées dans l'intervention. La question de la duplication se présente donc dès que l'on parle de recherche-action. Dans ce que Hélène Trocmé Fabre nomme une "pédagogie de l'incertitude", il faudra également gérer les transformations produites, les changements amorcés.

La recherche action est en quête de cohérence entre recherche et action. Ce n'est pas la tension entre les deux rôles du chercheur qui peut la lui donner. L'équilibre souhaité provient non des tensions, mais de l'articulation des tensions (en termes positifs) entretenues dans le système, et que lui confèrent les zones de liberté. C'est autour de notions comme la reliance ou les carrefours (Barbier, 1996) voire les frontières (Bazin, 2003) que se joue une possible évolution du système, toujours remise en question. La posture du chercheur lui permet de se positionner entre le pragmatisme de l'action et l'objectivité de la recherche menée avec rigueur.

Dans les propos recueillis auprès des enseignants en fin d'action-recherche, on constate qu'un petit nombre d'entre eux ont l'impression de n'avoir pas changé, même si leurs propos de départ sont en parfaite contradiction avec ceux de la fin de l'intervention et qu'ils ont objectivement évolué. La conscience du changement n'est pas acquise, le transfert s'est parfois effectué sans prise de conscience du déplacement. De manière symptomatique, un certain nombre évoque la compréhension des théories comme le premier indicateur d'apport de la recherche-action dans le cas des formations de type stages, mais pas dans celui des accompagnements d'équipes ou de personnes ni des suivis de projets, en revanche. Le second indicateur qu'ils signalent est celui des attitudes : ils ont développé un plaisir de..., un goût pour..., et une envie de..., souvent à titre personnel (de lire, de voyager, de se lancer dans l'informatique...) plus que professionnel. Vient ensuite le registre des pratiques et de leur évolution : ils ont fait... le plus souvent à partir d'exemples concrets. C'est également par la prise de conscience des valeurs qui sous- 
tendent l'action professionnelle que les enseignants manifestent leur compréhension du changement. Les élèves sont néanmoins les grands oubliés de ces constats.

On gagnera à l'avenir à s'outiller pour mieux observer les indicateurs du changement, afin d'apprendre à construire le changement et à le partager. Trois orientations se dessinent en ce sens :

- d'une part, il convient que les chercheurs structurent une vision des processus qui président au changement. Il est nécessaire de s'armer d'une méthodologie rigoureuse, qui ne s'arrête pas non plus avec la fin de l'action. En particulier on sera attentif à la gestion des traces, du temps, des chroniques de l'intervention dans la durée;

- d'autre part, il convient de trouver des interfaces pour lever la dichotomie entre recherche et action, permettre une traçabilité de l'intervention. Le terrain de la formation initiale étant particulièrement bien venu pour ce faire avec les formations de praticiens réflexifs, selon l'expression de Schön (Schön, 1987);

- enfin, il convient de questionner les représentations et les pratiques de tous les acteurs aux divers moments clés de la recherche-action et d'en vérifier la stabilisation, sans pour autant chercher à modifier les pratiques de ceux-ci. Le chercheur doit assumer ses responsabilités épistémologiques face aux praticiens. Et l'institution mieux accompagner les apports de ce type de recherche.

\section{Conclusion}

De fait, la richesse des approches de la recherche-action constitue sa force et signe apparemment de manière simultanée sa faiblesse. Elle vaut donc ici et maintenant, en lien fort avec ses contextes, pour les cas étudié. Elle tente d'approcher un idéal de cohérence et de transférabilité, qu'elle ne peut sans doute atteindre sans faire appel aux valeurs qui la portent. Il convient d'accepter la multiplicité des formes qu'elle prend au vu de sa souplesse de mise en œuvre dans un monde complexe.

Si la recherche-action a valeur de méthodologie de recherche parmi d'autres - et souvent en co-existence avec d'autres modalités de recherche, elle appelle pour une part la clarification des référents théoriques sous-jacents qui constituent les bases des modèles existants et pour une autre la rigueur des protocoles mettant en lien les situations et les visées du changement. Ce sont ces outils épistémologiques et idéologiques qui sont le ferment des pratiques professionnelles et de leur observation par la recherche-action.

\section{BIBLIOGRAPHIE}

Amado, G., Levy, A. (2001). La recherche-action : perspectives internationales. Paris : Eska.

Ardoino, J. (1966). Communications et relations humaines : esquisse d'un modèle d'intelligibilité des organisations. Institut d'organisation des entreprises de l'Université de Bordeaux.

Barbier, R. (1996). La recherche-action. Paris : Anthropos. 
Baribeau, C. (Ed.) (1992). La recherche-action de Kurt Lewin aux pratiques contemporaines, Revue de l'Association pour la recherche qualitative, numéro spécial, volume 7.

Bazin, H. (2003). Espaces populaires de création culturelle : enjeux d'une recherche-action situationnelle, http://www.recherche-action.fr/linkedDocuments/RA_29005_htm (lien valide à la date de publication).

Berbaum, J. (1982). Analyse systémique des actions de formation. Paris : PUF, collection Pédagogies d'aujourd'hui.

Blanchard-Laville, C. (et al.) (2003). Théoriser des pratiques professionnelles : intervention et rechercheaction en travail social. Paris : L'Harmattan, collection Savoir et formation.

Carr, W., Kemmis, S. (1986). Becoming Critical. Education, knowledge and action research. Lewes : Falmer Press.

Catroux, M. (2002). Introduction à la recherche-action : modalités d'une démarche théorique centrée sur la pratique. Les Cahiers de l'APLIUT, volume XXI, nº 3, mars 2002, pp. 9-19.

Charlier, B. (1998). Apprendre et changer sa pratique d'enseignement, expériences d'enseignants. Bruxelles : De Boeck Université, collection Pratiques pédagogiques.

Crézé, F., Liu, M. (2006). La recherche-action et les transformations sociales. Paris : L'Harmattan.

Ellis. R. (1997). SLA Research and Language Teaching. Oxford : Oxford University Press.

Gather Thurler, M. (2000). Innover au cœur de l'établissement scolaire. Paris : ESF.

Goyette, G., Lessart-Hebert, M. (1987). La recherche-action, ses fonctions, son fondement, son instrumentalisation. Québec : PUQ.

Guénard, D. (1991). Introduction à la dynamique léwinienne, 59² congrès de l'ACFAS. In : Baribeau, C. (Ed.) (1992). La recherche-action de Kurt Lewin aux pratiques contemporaines, Revue de l'Association pour la recherche qualitative, numéro spécial, volume 7.

Jonnaert, P., Van der Borght, C. (1999). Créer des conditions d'apprentissage- un cadre de référence socioconstructiviste pour une formation didactique des enseignants. Bruxelles : De Boeck Université, Collection Perspectives en éducation.

Kemmis, S. Mc Taggart, R. (1988). The Action Research Planner. Geelong, Victoria : Deakin University Press.

Lapassade, G. (1993). Ethnographie de l'école et recherche-action, université Paris VIII.

Levy, A. (1997). Sciences cliniques, organisation sociale. Paris : PUF.

Lewin, K. (1943). "Forces Behind Food Habits and Methods of Change". In Bulletin of National Resources Council, $\mathrm{n}^{\circ}$ 108. p. 35-65

Mc Taggart, R. (1996). Issues for participatory action researchers. In : O. Zuber-Skeritt (Ed.). New Directions in Action Research. London : Falmer Press.

Mesnier, P.-M., Missotte, P. (2003). La recherche-action, une autre manière de chercher, de se former, transformer. Paris : L'Harmattan.

Narcy-Combes, J.-P. (2005). Didactique des langues et TIC : vers une recherche-action responsable. Paris : Ophrys, collection Autoformation et enseignement multimédia.

Newman, J. (2000) Action Research : a brief Overview, Forum Qualitative Sozialforschung, Online Journal, http://qualitative-research.net (lien valide à la date de publication). 
Paquet, L, Altet, M., Charlier, E., Perrenoud, P. (eds.) (1996). Former des enseignants professionnels. Louvain La Neuve, De Boeck Université.

Schön, D. A. (1994). Le praticien réflexif. Montréal : Editions logiques.

Van der Maren, J.-M. (1999). La recherche appliquée en pédagogie. Bruxelles, De Boeck Université, Collection Méthodes en sciences humaines.

Vrancken, D., Kuty, O. (et al.) (2001). La sociologie de l'intervention : enjeux et perspectives. Bruxelles : De Boeck Université.

\section{Sites Internet}

Recherche-Action (2006). http://www.recherche-action.fr (lien valide à la date de publication).

Tele-Tandem (2006). http://www.tele-tandem.org (lien valide à la date de publication).

\section{NOTES}

1. Jack Lang alors ministre de l'Education nationale avait commandé une évaluation du dispositif français en matière d'apprentissage des langues à l'école élémentaire à un groupe de pilotage composé d'institutionnels de praticiens et de deux chercheurs. Des outils sont nés pour des tests. De ces outils sont nées les activités de Banqoutils, disponibles pour les classes de CM2 et de $3^{\mathrm{e}}$ et en ligne sur http://www.education.gouv.fr

2. DDL : didactique des langues.

3. Hugues Bazin reprend ici deux versions d'un texte de Lapassa de (1991-1993) intitulé : De l'ethnographie de l'école à la nouvelle recherche-action, Université de Paris VIII. Ces deux textes sont disponibles sur le site http://www.recherche-action.fr

4. La recherche-action n'est pas une méthode ou un protocole de recherche mais une série de convictions que l'on observe et problématise au travers de pratiques, une série de principes de conduite d'enquêtes sociales.

5. Pour une vision d'ensemble de la notion de recherche-action, on renverra par exemple à l'ouvrage de Goyette et Lessart-Hebert (Goyette et Lessart-Hebert, 1987)

6. Les approches anglo-saxonnes et anglo-américaines ont influencé un certain nombre de travaux de didacticiens des langues. Catroux, par exemple, souligne l'étonnante créativité du monde anglo-saxon pour des appellations fort diverses comme teacher research, classroom research, co-learning, cooperative enquiry, critical reflexion, ou encore practical enquiry (Catroux, 2002).

7. On entend ici par lieu classe, le groupe social de référence au sein d'un établissement scolaire qui travaille en un seul lieu ensemble.

\section{RÉSUMÉS}

La présente communication s'attache à examiner la notion de recherche-action et les débats qui la constituent dans le domaine de la didactique des langues. Il va s'agir de présenter une description et une interprétation de sa mise en œuvre. Pour ce faire, la réflexion s'appuiera sur des exemples rencontrés au cours de nombreuses années de formation d'enseignants de langues 
et d'accompagnement d'équipes et de projets innovants dans le cadre institutionnel de l'enseignement scolaire, ici volontairement limité au contexte français.

This talk examines the concept of action research, and the debates centred around it in the domain of foreign language teaching methodology. We will describe how action research is implemented, with illustrations taken from many years of experience training language teachers and accompanying teams and innovative projects in the school context. We will limit ourselves to a consideration of the French institutional setting.

\section{INDEX}

Mots-clés : didactique des langues, méthodologie de recherche, recherche-action, enseignement aux enfants

Keywords : foreign language teaching methodology, research methodology, action-research, young language learners

\section{AUTEUR}

\section{DOMINIQUE MONTAGNE-MACAIRE}

Dominique Montagne-Macaire est maître de conférences à l'IUFM d'Aquitaine Bordeaux, où elle coordonne les langues vivantes. Ses travaux portent sur les liens entre la didactique d'une langue et celle des langues, sur les dispositifs innovants dans le cadre institutionnel, sur l'enseignement des langues aux enfants et sur la formation des enseignants. Elle participe à la vie de l'Acedle (Association des chercheurs et enseignants didacticiens des langues étrangères) depuis sa création. Elle est membre de l'équipe du Diltec (Didactique des langues, des textes et des cultures), Université Paris 3 (EA 2288).

Courriel : dominique.macaire[at]aquitaine.iufm.fr Adresse : IUFM d'Aquitaine, Château Bourran, BP 152, 160 avenue de Verdun, F-33705 Merignac. 